\title{
Gestión del conocimiento en la micro y pequeña empresa mexicana de la industria del software
}

\author{
José Sergio Ruiz Castilla ${ }^{1}$, Yulia Ledeneva ${ }^{2}$, Héctor Cuesta Arvizu ${ }^{1}$ \\ ${ }^{1}$ Centro Universitario UAEM Texcoco \\ Texcoco, Estado de México, México \\ ${ }^{2}$ Unidad Académica Profesional Tianguistenco, \\ Tianguistenco, Estado de México, México
}

jsergioruizc@gmail.com,yledeneva@yahoo.com,hm_cuesta@yahoo.com.mx

\begin{abstract}
Resumen. Las empresas que desarrollan software en México son microempresas con diez o menos empleados que no cuentan con sistemas de gestión del conocimiento. Se parte de un modelo de transferencia de conocimiento de los desarrolladores expertos a los no expertos, a través de un sistema de gestión del conocimiento. Se enfocó la investigación en conocer ¿Cómo sucede la gestión de conocimiento en las micro y pequeñas empresas que desarrollan software en México? Se encontró que el problema no es solo tecnológico, sino también cultural, en dichas organizaciones. Por lo anterior se diseñó un instrumento para medir la cultura de la gestión del conocimiento, se aplicó y se muestran los resultados. Se detectó que los desarrolladores consideran muy importante compartir el conocimiento y de hecho lo hacen informalmente. Se concluyó que dichas organizaciones deben incluir la gestión del conocimiento en sus procesos de desarrollo de software.
\end{abstract}

Palabras clave: Conocimiento, gestión del conocimiento, base de conocimiento, activos de conocimiento, compartir conocimiento, industria del software.

\section{Introducción}

En México la industria del software se concentra en el 1.5\% de empresas con 100 empleados o más, mientras que el 78.5\% son MiyPEDS (Micros y Pequeñas Empresas Desarrolladoras de Software) con menos de 10 empleados [4], además de la concentración de las 2853 empresas en 5 estados de la República Mexicana; en el Distrito Federal con el 28\%, Nuevo León con el 11\%, Jalisco con el $8 \%$, el Estado de México con el 6\%, Puebla con el 4\% y el $43 \%$ en los demás estados del país [4].

El objetivo de este trabajo de investigación es conocer la GC (Gestión del Conocimiento) en las MiyPEDS, ofreciendo resultados obtenidos a partir de la aplicación de un instrumento de medición de diversos aspectos de la GC buscando hacer una aportación para las propias MiyPEDS. 
El desarrollo del conocimiento con sentido de equidad social identifica y pondera las necesidades, intereses y demandas de grupos sociales que son beneficiados por el desarrollo científico y tecnológico. Por lo anterior se proponen "redes socio-culturales de innovación" formada por expertos diversos para producir conocimiento [12].

El conocimiento en las PyMEs (Pequeñas y Medianas Empresas) desarrolladoras de software mantienen conocimiento tácito en la mente de sus trabajadores y conocimiento explícito en: sus proceso, sistemas, manuales, patentes, y otros medios; sin embargo deben valorar que la gestión del conocimiento puede tener una influencia positiva y fomentadora de los niveles de productividad y competitividad.

Los trabajadores son los poseedores del conocimiento, habilidades, destrezas y experiencias; Así lo demuestran los resultados de la influencia que tiene la gestión del conocimiento en el nivel de competitividad de las PyMEs manufactureras de Aguascalientes [5].

Los SBC (Sistemas Basados en el Conocimiento) también son un factor importante en la solución de problemas. En la industria del vestido por ejemplo es posible mejorar el diseño usando el conocimiento de expertos y un SBC para obtener resultados más óptimos en la utilización de los recursos materiales [11].

Las empresas deben aprender y capitalizar el conocimiento. Para aprender se requiere que se comparta el conocimiento que puede ser en comunidades de prácticas y de aprendizaje. Generar activos de conocimiento y compartirlos dentro de la organización. En medida que la organización aprenda promueve la cultura de la gestión del conocimiento [6].

Para lograr la adquisición del conocimiento y capital intelectual en la empresa de manufactura en México es necesario identificar cuáles son los activos intangibles de la propia empresa y de otras organizaciones que guardan similitudes para buscar y lograr la transferencia de conocimiento. Las organizaciones deben de contar con su base de conocimiento para almacenar sus mejores prácticas, procesos e innovaciones. La gestión del conocimiento concluirá con una creación de valor para la empresa [6].

Los activos tangibles son conocimientos explícitos en medios impresos o digitales; mientras que los intangibles se refieren al conocimiento tácito que está en la mente de los desarrolladores. En las MiyPEDs se carece de recursos, pero también de cultura para llevar a cabo la gestión del conocimiento. Existe la necesidad de contar con modelos y recursos para llevar a cabo gestión del conocimiento en dichas organizaciones. La gestión del conocimiento en las MiyPEDs deben tener la misión de la mejora en los procesos [1].

\section{Gestión del conocimiento}

En el trabajo de Ruiz se presentó un modelo para la gestión del conocimiento (Fig. 1). En dicho modelo se busca promover la GC en la industria del software en México [10].

El modelo de gestión de conocimiento es parte de un proyecto que busca establecer primero un modelo y una plataforma para un SGC (Sistema de Gestión del Conocimiento) así como proponer esquemas para el fomento de la GC en las MiyPEDS en México. 


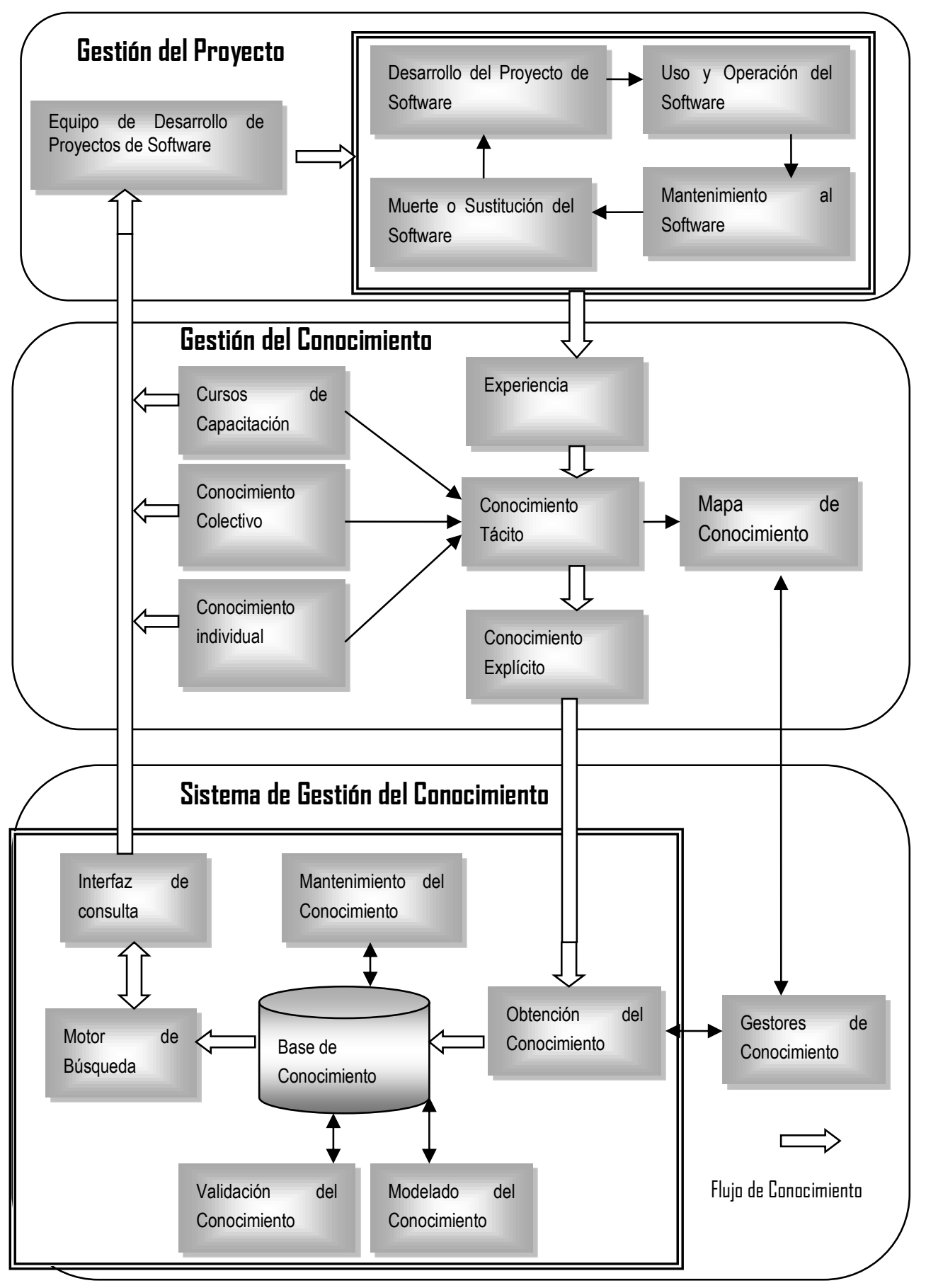

Fig. 1. Modelo de Gestión del conocimiento para el desarrollo de software (Ruizset al., 201Rebearch in Computing Science 73 (2014) 
El modelo cuenta con tres componentes: el primero la gestión de proyectos donde se genera experiencia al desarrollar los proyectos. El segundo la GC donde el conocimiento tácito se origina a partir de la experiencia al desarrollar proyectos de software, de los demás elementos del equipo de trabajo, de los cursos de capacitación y del conocimiento adquirido durante su formación. El conocimiento tácito se debe transformar en conocimiento explícito susceptible de almacenamiento en una base de conocimiento. El tercero es el SGC, donde se requiere que el conocimiento explícito debe obtenerse, almacenarse, validarse, mantenerse y consultarse por los usuarios no expertos. Lo anterior con el fin de reutilizar el conocimiento y mejorar los procesos de desarrollo de software. Es importante en los SGC se consulte y use el conocimiento, de lo contrario se corre el riesgo de crear un cementerio de conocimiento [10].

\subsection{Actividad de compartir el conocimiento en las MiyPEDS en México}

Las lecciones aprendidas se obtienen al culminar una tarea, actividad o un proyecto, las cuales se deben documentar para una consulta posterior por todos los integrantes de la organización [8]. En México en el modelo MoProSoft (Modelos de Procesos para la industria del software) recomienda almacenar la documentación de los procesos en una base de conocimiento de acceso libre para los integrantes de la organización [8].

Compartir el conocimiento en las organizaciones puede ser una ventaja competitiva que permite agregar valor a los productos y servicios y a la organización misma. Sin embargo para compartir el conocimiento es necesario un ambiente, herramientas y procesos definidos para dicha tarea [2].

El conocimiento se crea todos los días en la organización y crece como conocimiento tácito en la mente de cada uno de los miembros de la organización, pero es mejor si se comparte lo que se sabe con los demás.

El conocimiento se genera y se acumula a partir de lo que sé, de lo que aprendo de otros y lo que aprendo haciendo. Una vez que se posee se debe compartir con los demás logrando un conocimiento colectivo u organizacional [10].

La actividad de compartir conocimiento, debe de premiarse con estímulos económicos y no económicos que motiven a los trabajadores del conocimiento a seguir aportando conocimiento.

Compartir el conocimiento de persona a persona requiere de un ambiente adecuado, pero en organizaciones distribuidas o grandes es necesaria una estrategia para trasformar el conocimiento tácito a explícito y de explícito a tácito [7].

\subsection{Transferencia del conocimiento en las MyPEDS en México}

En México la transferencia sucede a través de cursos de capacitación, de cara a cara y a través de internet; sin embargo los desarrolladores lo hacen informalmente, porque no existen procesos y políticas que lo incluyan como actividad necesaria en el desarrollo de software.

Vale la pena conocer cómo se transfiere el conocimiento en otras organizaciones y culturas, para contrastar con la cultura de la transferencia del conocimiento en México. La transferencia del conocimiento suele darse primeramente cara a cara en 
las expendedoras de agua, salas de descanso y comedores, además de espacios destinados para ello como ferias de conocimiento y foros abiertos, dónde las personas socializan. Se trata de una nueva forma de pensar, en IBM en algún momento se informó a los trabajadores iDejen de conversar y pónganse a trabajar! cuando se requiere de ;Comiencen a conversar y pónganse a trabajar! En el caso de los japoneses pasan muchas horas reunidos después del trabajo con el fin de socializar y transferir el conocimiento. La segunda forma es a través de documentos, repositorios de documentos, la intranet, groupware y SGC. Y la tercera forma consiste en aprender viendo, se dispone de un experto y un aprendiz que al observar cómo se desarrollan las actividades aprende [3].

Es posible trabajar con grupos de trabajo con un moderador donde se busque la socialización y la transferencia de conocimiento tácito [2]. Es importante que los empleados coincida en el mismo sitio, como comedores, áreas de café, salas de juntas y así tener contacto entre ellos y dialoguen, solo así se puede promover la transferencia del conocimiento. La socialización es el medio más eficaz para la transferencia del conocimiento tácito-tácito, por eso en Japón se acostumbra que las personas socialicen, de hecho prefieren la transferencia del conocimiento tácito [7].

\subsection{Almacenamiento del conocimiento}

Es importante obtener un inventario de activos de conocimiento, que corresponden al conjunto de conocimientos, que posee una persona o plasmado en un medio y que guarda de forma cohesiva la solución de un problema específico, dentro de un dominio del conocimiento.

Los activos de conocimiento tácito podrán existir en la mente de los desarrolladores, y se generan nuevos activos de conocimiento a medida que el desarrollador tiene nuevas experiencias u obtiene conocimientos de otros desarrolladores.

¿Dónde almacenar los activos de conocimiento? En la empresa se guardan los activos de conocimiento explícito en una base de conocimiento; dichos activos de conocimiento explícito aumentarán en medida que se introduzcan a la base de conocimiento.

Finalmente el conocimiento de la empresa debe considerar el conocimiento tácito del capital humano y los activos de conocimiento explícito de la base de conocimiento.

\subsection{Inventario del conocimiento}

El inventario de conocimiento consiste en identificar cada activo de conocimiento y registrarlo en la base de conocimiento. ¿Existe redundancia de conocimiento? Sí. Cuándo dos o más desarrolladores poseen el conocimiento. En el caso anterior solo aparecerá una vez en la base de conocimiento. En ese caso son dos poseedores, pero un solo activo de conocimiento. El sistema de almacenamiento de conocimiento no debe permitir que se dupliquen los activos de conocimiento.

Para el almacenamiento del conocimiento es necesario un repositorio de conocimiento denominado base de conocimiento donde se almacenan: archivos de texto, de audio o de video. ¿Quiénes debe administrar el conocimiento? Será 
necesario destinar personas que administrarán el conocimiento que pueden ser gestores o gerentes de la GC. El conocimiento almacenado forma parte de un inventario de conocimiento. La unidad son componentes de conocimientos llamados activos de conocimiento $[3,6]$.

Otro nombre que se le ha dado al repositorio de conocimiento ha sido sedes del conocimiento, de igual manera tiene la misión de determinar dónde está el conocimiento, en este caso puede ser un sistema Web con acceso a los usuarios para la búsqueda y consulta del conocimiento [2].

\section{Método utilizado}

\subsection{Modelo para la gestión del conocimiento}

Tomando como base el modelo de conocimiento de la Figura 1. Permite establecer cómo se genera, transforma, almacena, administra y utiliza el conocimiento en la industria del software.

\subsection{Cultura de la gestión del conocimiento}

Se diseñó un instrumento que permitiera aplicarse a desarrolladores de MyPEDS y conocer la cultura de la gestión del conocimiento. Cuenta con 50 preguntas que el desarrollador puede contestar con una elección. Se dividió en 11 secciones que se muestran en la Tabla 1. Para medir su comportamiento, posición y opinión. Se aplicó la siguiente escala: totalmente de acuerdo, de acuerdo, ni de acuerdo ni en desacuerdo, en desacuerdo y totalmente en desacuerdo.

Tabla 1. Secciones del instrumento de medición de la cultura de la gestión.

\begin{tabular}{ll}
\hline \multicolumn{3}{c}{ Secciones del instrumento } \\
\hline $\mathbf{1}$ & Actividad de compartir el conocimiento \\
$\mathbf{2}$ & Con quienes se comparte el conocimiento \\
$\mathbf{3}$ & Valor del conocimiento \\
$\mathbf{4}$ & Razones por la que no se comparte el conocimiento \\
$\mathbf{5}$ & Ventajas de compartir el conocimiento \\
$\mathbf{6}$ & Pérdida de conocimiento al convertir el conocimiento tácito a explícito \\
$\mathbf{7}$ & Pago y recompensa por compartir el conocimiento \\
$\mathbf{8}$ & Disposición para consultar el conocimiento \\
$\mathbf{9}$ & Medios para la transferencia del conocimiento \\
$\mathbf{1 0}$ & Cultura de la gestión del conocimiento \\
$\mathbf{1 1}$ & Tiempo disponible para la consulta del conocimiento \\
\hline
\end{tabular}

Las respuestas dependen de las políticas y prácticas de la empresa, así como de la forma de pensar y actuar de los desarrolladores. Con el instrumento se buscó conocer 
si los desarrolladores llevan a cabo prácticas de gestión y compartir el conocimiento. Se agregó un apartado para saber que esperan como pago o recompensa por compartir el conocimiento. Se intentó además conocer su posición para llevar a cabo la gestión del conocimiento si la empresa lo requiere. En este caso se aplicó el instrumento en el estado de Michoacán en una empresa dedicada al desarrollo de software. La empresa tienen sus clientes principalmente del ramo financiero en México D. F. Desarrollan software a medida de las necesidades de las organizaciones, soporte técnico y hosting de bases de datos. Cuentan con un turno de 9:00 a 18:00 horas de lunes a sábado y trabajan tanto hombres como mujeres.

\section{Resultados}

Una vez aplicado el instrumento se obtuvieron datos que se procesaron y se muestran resultados en las tablas siguientes.

Lo primero que se preguntó fue la posición de los desarrolladores acerca de compartir el conocimiento con otros desarrolladores, como desarrollador de una organización o como independiente, Tabla 2.

Tabla 2. Actividad de compartir el conocimiento.

\begin{tabular}{llll}
\hline \multicolumn{2}{c}{ Actividad de compartir el conocimiento } & & \\
\hline & $\begin{array}{l}\text { ¿Cuál es su } \\
\text { posición en } \\
\text { relación a } \\
\text { compartir su } \\
\text { conocimiento con } \\
\text { otros }\end{array}$ & $\begin{array}{l}\text { ¿Es más factible } \\
\text { compartir el } \\
\text { conocimiento } \\
\text { cuando se trabaja } \\
\text { en una } \\
\text { organización? }\end{array}$ & $\begin{array}{l}\text { ¿Es más factible } \\
\text { compartir el } \\
\text { conocimiento } \\
\text { cuando se } \\
\text { trabaja en forma } \\
\text { independiente? }\end{array}$ \\
& $\mathbf{9 2 . 0 \%}$ & $\mathbf{4 6 . 0 \%}$ & $12.5 \%$ \\
Totalmente de acuerdo & $8.0 \%$ & $42.0 \%$ & $4.2 \%$ \\
De acuerdo & $0.0 \%$ & $12.0 \%$ & $\mathbf{4 5 . 8 \%}$ \\
Ni de acuerdo, ni en desacuerdo & $0.0 \%$ & $12.5 \%$ \\
En desacuerdo & $0.0 \%$ & $0.0 \%$ & $25.0 \%$ \\
Totalmente en desacuerdo & $0.0 \%$ & $100.0 \%$ & $100.0 \%$ \\
\hline
\end{tabular}

De acuerdo a los resultados el $100 \%$ está de acuerdo en compartir el conocimiento. El 88\% Perciben más factible compartir el conocimiento dentro de una organización, mientras que las opiniones se dividen cuando trabajan de forma independiente.

Se les cuestionó con quien están de acuerdo en compartir el conocimiento, considerando sus colegas y compañeros de equipo de trabajo, Tabla 3. 
Tabla 3. Con quien compartir el conocimiento.

\begin{tabular}{lllll}
\hline \multicolumn{5}{c}{ Con quien compartir el conocimiento } \\
& $\begin{array}{l}\text { El conocimiento no se debe } \\
\text { compartir }\end{array}$ & $\begin{array}{l}\text { Compa } \\
\text { rtir su } \\
\text { trabajo } \\
\text { con su } \\
\text { equipo } \\
\text { de } \\
\text { trabajo }\end{array}$ & $\begin{array}{l}\text { Compartir } \\
\text { conocimie } \\
\text { nto en sus } \\
\text { empresa }\end{array}$ & $\begin{array}{l}\text { Compartir } \\
\text { conocimie } \\
\text { nto con } \\
\text { cualquier } \\
\text { desarrolla } \\
\text { dor }\end{array}$ \\
& & $12.5 \%$ & $\mathbf{2 5 . 0 \%}$ & $\mathbf{4 5 . 5 \%}$ \\
& $0.0 \%$ & $0.0 \%$ & $\mathbf{2 5 . 0 \%}$ & $18.2 \%$ \\
Totalmente de acuerdo & $8.3 \%$ & $16.7 \%$ & $20.8 \%$ & $27.3 \%$ \\
$\begin{array}{l}\text { Ne acuerdo } \\
\text { Ni de acuerdo, ni en }\end{array}$ & $0.0 \%$ & $0.0 \%$ & $8.4 \%$ & $4.5 \%$ \\
$\begin{array}{l}\text { En desacuerdo } \\
\begin{array}{l}\text { Totalmente en } \\
\text { desacuerdo }\end{array}\end{array}$ & $\mathbf{9 1 . 7 \%}$ & $\mathbf{7 0 . 8 \%}$ & $20.8 \%$ & $4.5 \%$ \\
& $100.0 \%$ & $100.0 \%$ & 100.0 & 100.0 \\
\hline
\end{tabular}

Los resultados indican que el $91.7 \%$ refieren que no están de acuerdo en no compartir el conocimiento. Mientras que el $70.8 \%$ no están de acuerdo en compartir el conocimiento solo con sus compañeros. A el 50\% le interesa compartir a nivel de toda la organización y el $63.7 \%$ con cualquier otro desarrollador.

Otro cuestionamiento fue acerca del valor del conocimiento que le dan los desarrolladores para sí mismos, para su empresa, la industria y el país, Tabla 4.

Tabla 4. Valor del conocimiento.

\begin{tabular}{|c|c|c|c|c|}
\hline \multicolumn{5}{|c|}{ Valor del conocimiento } \\
\hline & $\begin{array}{l}\text { El } \\
\text { conocimient } \\
\text { o tiene alto } \\
\text { valor para } \\
\text { las personas }\end{array}$ & $\begin{array}{l}\text { El } \\
\text { conocimiento } \\
\text { tiene alto } \\
\text { valor para las } \\
\text { organizacione } \\
\mathrm{s}\end{array}$ & $\begin{array}{l}\text { El } \\
\text { conocimient } \\
\text { o tiene alto } \\
\text { valor para la } \\
\text { industria del } \\
\text { software }\end{array}$ & $\begin{array}{l}\text { El } \\
\text { conocimient } \\
\text { o tiene alto } \\
\text { valor para la } \\
\text { economía } \\
\text { del país }\end{array}$ \\
\hline Totalmente de acuerdo & $91.6 \%$ & $95.8 \%$ & $91.3 \%$ & $95.7 \%$ \\
\hline De acuerdo & $4.2 \%$ & $0.0 \%$ & $8.7 \%$ & $4.3 \%$ \\
\hline $\begin{array}{l}\mathrm{Ni} \text { de acuerdo, ni en } \\
\text { desacuerdo }\end{array}$ & $4.2 \%$ & $4.2 \%$ & $0.0 \%$ & $0.0 \%$ \\
\hline En desacuerdo & $0.0 \%$ & $0.0 \%$ & $0.0 \%$ & $0.0 \%$ \\
\hline \multirow[t]{2}{*}{ Totalmente en desacuerdo } & $0.0 \%$ & $0.0 \%$ & $0.0 \%$ & $0.0 \%$ \\
\hline & $100.0 \%$ & $100.0 \%$ & $100.0 \%$ & $100.0 \%$ \\
\hline
\end{tabular}

El $95.8 \%$ de los desarrolladores están de acuerdo en que el conocimiento tiene un alto valor para sí mismos y su empresa y el $100 \%$ está de acuerdo en que tiene un alto valor para la industria del software y para el país. Se les preguntó también por las 
razones por las que no se comparte el conocimiento y de la propiedad del conocimiento Tabla 5.

Tabla 5. Razones por las que no se comparte el conocimiento.

\begin{tabular}{|c|c|c|c|c|}
\hline \multicolumn{5}{|c|}{ Razones por las que no se comparte el conocimiento } \\
\hline & $\begin{array}{l}\text { Porque es } \\
\text { personal }\end{array}$ & $\begin{array}{l}\text { Porque es de } \\
\text { la } \\
\text { organización }\end{array}$ & $\begin{array}{l}\text { Porque es } \\
\text { estratégico } \\
\text { para la } \\
\text { organización }\end{array}$ & $\begin{array}{l}\text { Porque es } \\
\text { propiedad del } \\
\text { desarrollador }\end{array}$ \\
\hline Totalmente de acuerdo & $0.0 \%$ & $21.7 \%$ & $\mathbf{5 0 . 0 \%}$ & $4.5 \%$ \\
\hline De acuerdo & $0.0 \%$ & $17.4 \%$ & $16.7 \%$ & $9.1 \%$ \\
\hline $\begin{array}{l}\text { Ni de acuerdo, ni en } \\
\text { desacuerdo }\end{array}$ & $12.5 \%$ & $17.4 \%$ & $8.3 \%$ & $9.1 \%$ \\
\hline En desacuerdo & $12.5 \%$ & $8.7 \%$ & $12.5 \%$ & $45.5 \%$ \\
\hline \multirow[t]{2}{*}{ Totalmente en desacuerdo } & $\mathbf{7 5 . 0 \%}$ & $34.8 \%$ & $12.5 \%$ & $31.8 \%$ \\
\hline & $100.0 \%$ & $100.0 \%$ & $100.0 \%$ & $100.0 \%$ \\
\hline
\end{tabular}

El $87.5 \%$ de los desarrolladores cree que el conocimiento es personal y el $43 \%$ considera que es de la organización, con opiniones muy divididas. Por lo tanto coinciden que el conocimiento es de la organización aun cuando lo poseen los trabajadores.

Se incluyó un apartado para conocer qué ventajas avizoran al compartir el conocimiento al dar y recibir conocimiento Tabla 6.

Tabla 6. Ventajas de compartir el conocimiento.

\begin{tabular}{lllll}
\hline \multicolumn{5}{c}{ Ventajas de compartir el conocimiento } \\
& $\begin{array}{l}\text { En posible } \\
\text { reutilizar el } \\
\text { conocimient } \\
\text { o }\end{array}$ & $\begin{array}{l}\text { Que otros } \\
\text { desarrolladore } \\
\text { s conozcan y } \\
\text { usen su } \\
\text { conocimiento }\end{array}$ & $\begin{array}{l}\text { En } \\
\text { aprender } \\
\text { de otros } \\
\text { a }\end{array}$ & $\begin{array}{l}\text { Permite } \\
\text { productividad } \\
\text { en las } \\
\text { organizacione } \\
\text { s }\end{array}$ \\
& $\mathbf{9 1 . 7 \%}$ & $\mathbf{7 4 . 0 \%}$ & $\mathbf{7 9 . 2}$ & $\mathbf{8 3 . 3 \%}$ \\
Totalmente de acuerdo & $8.3 \%$ & $13.0 \%$ & $20.8 \%$ & $16.7 \%$ \\
$\begin{array}{l}\text { De acuerdo } \\
\text { Ni de acuerdo, ni en } \\
\text { desacuerdo }\end{array}$ & $0.0 \%$ & $13.0 \%$ & $0.0 \%$ & $0.0 \%$ \\
En desacuerdo & $0.0 \%$ & $0.0 \%$ & $0.0 \%$ & $0.0 \%$ \\
Totalmente en desacuerdo & $0.0 \%$ & $0.0 \%$ & $0.0 \%$ & $0.0 \%$ \\
& $100 \%$ & $100 \%$ & $100 \%$ & $100 \%$ \\
\hline
\end{tabular}

Todos están convencidos que el conocimiento se puede reutilizar. El $87 \%$ está dispuesto a compartir el conocimiento hacia otros. Todos están de acuerdo en que 
pueden aprender de otros y que existe mayor productividad en la organización al compartir el conocimiento.

Se preguntó acerca de la pérdida de conocimiento que existe cuando exteriorizan el conocimiento tácito con el fin de convertirlo en explícito en algún medio, Tabla7.

Tabla 7. Pérdida de conocimiento al convertirlo de tácito a explícito.

\begin{tabular}{lllll}
\hline \multicolumn{4}{c}{ Pérdida de conocimiento al convertirlo de tácito a explícito } \\
\hline & $\begin{array}{l}\text { No sabe cómo } \\
\text { documentar su } \\
\text { conocimiento }\end{array}$ & $\begin{array}{l}\text { No desea } \\
\text { revelar los } \\
\text { detalles del } \\
\text { conocimiento }\end{array}$ & $\begin{array}{l}\text { Existen } \\
\text { aspectos } \\
\text { imposibles } \\
\text { de } \\
\text { explicar }\end{array}$ & $\begin{array}{l}\text { No sabe } \\
\text { cómo } \\
\text { exponer sus } \\
\text { experiencias }\end{array}$ \\
Totalmente de acuerdo & $4.1 \%$ & $0.0 \%$ & $12.5 \%$ & $0.0 \%$ \\
De acuerdo & $16.7 \%$ & $4.2 \%$ & $20.8 \%$ & $\mathbf{2 9 . 2 \%}$ \\
Ni de acuerdo, ni en desacuerdo & $\mathbf{4 1 . 7 \%}$ & $16.6 \%$ & $25.0 \%$ & $20.8 \%$ \\
En desacuerdo & $12.5 \%$ & $12.5 \%$ & $8.4 \%$ & $\mathbf{2 9 . 2 \%}$ \\
Totalmente en desacuerdo & $25.0 \%$ & $\mathbf{6 6 . 7 \%}$ & $\mathbf{3 3 . 3 \%}$ & $20.8 \%$ \\
& $100.0 \%$ & $100.0 \%$ & $100.0 \%$ & $100.0 \%$ \\
\hline
\end{tabular}

No están de acuerdo y desconocen el proceso de convertir el conocimiento, se asume que lo hacen, pero no tienen claro cómo. Mientras que el $79.2 \%$ no desean hacerlo.

Se cuestionó el pago por el conocimiento para conocer si están de acuerdo en donar o vender su conocimiento tácito y explícito, Tabla 8.

Tabla 8. Pago y recompensas por el conocimiento.

\begin{tabular}{lclll}
\hline \multicolumn{5}{c}{ Pago y recompensas por el conocimiento } \\
\hline & $\begin{array}{l}\text { ¿Compartiría su } \\
\text { conocimiento } \\
\text { sin pago y } \\
\text { reconocimientos } \\
?\end{array}$ & $\begin{array}{l}\text { ¿Compartiría } \\
\text { su } \\
\text { conocimiento } \\
\text { por algún } \\
\text { reconocimient } \\
\text { o no } \\
\text { monetario? }\end{array}$ & $\begin{array}{l}\text { ¿ su } \\
\text { conocimient } \\
\text { o por un } \\
\text { bono? }\end{array}$ & $\begin{array}{l}\text { ¿Compartíí } \\
\text { a su } \\
\text { conocimient } \\
\text { o por un } \\
\text { pago en } \\
\text { efectivo? }\end{array}$ \\
$\begin{array}{l}\text { Totalmente de acuerdo } \\
\text { De acuerdo }\end{array}$ & $\mathbf{5 0 . 0 \%}$ & $\mathbf{6 2 . 5 \%}$ & $\mathbf{5 0 . 0 \%}$ & $\mathbf{3 7 . 5 \%}$ \\
Ni de acuerdo, ni en & $29.1 \%$ & $12.5 \%$ & $12.5 \%$ & $8.3 \%$ \\
desacuerdo & $12.5 \%$ & $16.6 \%$ & $16.6 \%$ & $29.2 \%$ \\
Totalmente en desacuerdo & $4.2 \%$ & $4.2 \%$ & $4.2 \%$ & $4.2 \%$ \\
& $4.2 \%$ & $4.2 \%$ & $16.7 \%$ & $20.8 \%$ \\
& $100.0 \%$ & $100.0 \%$ & $100.0 \%$ & $100.0 \%$ \\
\hline
\end{tabular}

Los resultados resultaron interesantes porque existe la voluntad de donar el conocimiento por parte del $79.1 \%$ pero el $75 \%$ esperan reconocimientos y el $62.5 \%$ un bono y solo el $45.8 \%$ esperan un pago en efectivo. 
También se incluyeron preguntas de la disposición que tienen para consultar el conocimiento, si existiere en la organización, Tabla 9.

Tabla 9. Disposición para consultar el conocimiento.

\begin{tabular}{|c|c|c|c|c|}
\hline \multicolumn{5}{|c|}{ Disposición para consultar el conocimiento } \\
\hline & $\begin{array}{l}\text { Le interesa } \\
\text { consultar el } \\
\text { conocimient } \\
\text { o de otros }\end{array}$ & $\begin{array}{l}\text { Consultaría } \\
\text { conocimiento } \\
\text {, solo si es } \\
\text { política de la } \\
\text { organización }\end{array}$ & $\begin{array}{l}\text { Mientras } \\
\text { desarrolla, si } \\
\text { consulta } \\
\text { conocimient } \\
\text { o }\end{array}$ & $\begin{array}{l}\text { Siempre } \\
\text { consulta } \\
\text { conocimient } \\
\text { o }\end{array}$ \\
\hline Totalmente de acuerdo & $81.8 \%$ & $8.7 \%$ & $76.2 \%$ & $43.5 \%$ \\
\hline De acuerdo & $13.6 \%$ & $4.4 \%$ & $19.0 \%$ & $13.0 \%$ \\
\hline $\begin{array}{l}\text { Ni de acuerdo, ni en } \\
\text { desacuerdo }\end{array}$ & $4.6 \%$ & $21.7 \%$ & $4.8 \%$ & $34.8 \%$ \\
\hline En desacuerdo & $0.0 \%$ & $21.7 \%$ & $0.0 \%$ & $8.7 \%$ \\
\hline \multirow[t]{2}{*}{ Totalmente en desacuerdo } & $0.0 \%$ & $43.5 \%$ & $0.0 \%$ & $0.0 \%$ \\
\hline & $100.0 \%$ & $100.0 \%$ & $100.0 \%$ & $100.0 \%$ \\
\hline
\end{tabular}

Se encontró que el $95.4 \%$ les interesa el conocimiento de otros. El $65.2 \%$ opina que no es necesaria una política de la organización. Es importante notar que el $95.2 \%$ consulta conocimiento, pero otro $56.5 \%$ siempre lo hace.

Se incluyeron preguntas para conocer los medios conde almacenan y comparten el conocimiento, Tabla 10.

Tabla 10. Medios para la transferencia del conocimiento.

\begin{tabular}{|c|c|c|c|c|}
\hline \multicolumn{5}{|c|}{ Medios para la transferencia del conocimiento } \\
\hline & $\begin{array}{l}\text { Prefiere } \\
\text { cursos para } \\
\text { obtener } \\
\text { nuevos } \\
\text { conocimiento } \\
\text { s }\end{array}$ & $\begin{array}{l}\text { Prefiere } \\
\text { manuales } \\
\text { para obtener } \\
\text { nuevos } \\
\text { conocimiento } \\
\text { s }\end{array}$ & $\begin{array}{l}\text { Prefiere } \\
\text { videos para } \\
\text { obtener } \\
\text { nuevos } \\
\text { conocimiento } \\
\text { s }\end{array}$ & $\begin{array}{l}\text { Prefiere un } \\
\text { sistema de } \\
\text { gestión del } \\
\text { conocimient } \\
\text { o }\end{array}$ \\
\hline Totalmente de acuerdo & $37.5 \%$ & $25.0 \%$ & $33.3 \%$ & $34.8 \%$ \\
\hline De acuerdo & $33.3 \%$ & $33.3 \%$ & $29.2 \%$ & $52.2 \%$ \\
\hline $\begin{array}{l}\text { Ni de acuerdo, ni en } \\
\text { desacuerdo }\end{array}$ & $20.8 \%$ & $29.2 \%$ & $37.5 \%$ & $8.7 \%$ \\
\hline En desacuerdo & $4.2 \%$ & $8.3 \%$ & $0.0 \%$ & $4.3 \%$ \\
\hline \multirow[t]{2}{*}{ Totalmente en desacuerdo } & $4.2 \%$ & $4.2 \%$ & $0.0 \%$ & $0.0 \%$ \\
\hline & 100.0 & $100.0 \%$ & $100.0 \%$ & $100.0 \%$ \\
\hline
\end{tabular}

De acuerdo las opiniones aceptan los diferentes formatos mencionados para la transferencia del conocimiento. El $70.8 \%$ prefieren cursos, el $58.3 \%$ manuales, $62.5 \%$ prefieren videos y el $87 \%$ prefieren un SGC.

Se incluyeron las preguntas más importantes para conocer las actividades de la gestión del conocimiento para conocer la cultura de la GC, Tabla 11. 
Tabla 11. Cultura de la gestión del conocimiento.

\begin{tabular}{|c|c|c|c|c|}
\hline \multicolumn{5}{|c|}{ Cultura de la Gestión del conocimiento } \\
\hline & $\begin{array}{l}\text { ¿Genera un } \\
\text { repositorio } \\
\text { de su } \\
\text { conocimient } \\
\text { o? }\end{array}$ & $\begin{array}{l}\text { ¿Consulta su } \\
\text { repositorio } \\
\text { de } \\
\text { conocimient } \\
o \text { ? }\end{array}$ & $\begin{array}{l}\text { ¿Comparte } \\
\text { el } \\
\text { conocimient } \\
\text { o de su } \\
\text { repositorio? }\end{array}$ & $\begin{array}{l}\text { ¿Consulta el } \\
\text { conocimiento } \\
\text { de otros } \\
\text { desarrolladore } \\
\text { s? }\end{array}$ \\
\hline Totalmente de acuerdo & $17.4 \%$ & $\mathbf{3 4 . 8 \%}$ & $56.5 \%$ & $68.2 \%$ \\
\hline De acuerdo & $34.8 \%$ & $26.1 \%$ & $21.8 \%$ & $22.8 \%$ \\
\hline $\begin{array}{l}\mathrm{Ni} \text { de acuerdo, ni en } \\
\text { desacuerdo }\end{array}$ & $30.4 \%$ & $13.0 \%$ & $13.0 \%$ & $4.5 \%$ \\
\hline En desacuerdo & $13.0 \%$ & $17.4 \%$ & $8.7 \%$ & $4.5 \%$ \\
\hline \multirow[t]{2}{*}{ Totalmente en desacuerdo } & $4.4 \%$ & $8.7 \%$ & $0.0 \%$ & $0.0 \%$ \\
\hline & $100.0 \%$ & $100.0 \%$ & $100.0 \%$ & $100.0 \%$ \\
\hline
\end{tabular}

Se encontró que el $52.2 \%$ tiene una repositorio de conocimiento, que el $60.9 \%$ lo consulta, el $78.3 \%$ comparte su repositorio y el $91 \%$ consulta el conocimiento de otros.

Se incluyeron preguntas para conocer el tiempo que disponen y estarían dispuestos a dedicar a la gestión del conocimiento, Tabla 12.

Tabla 12. Tiempo disponibles para la gestión del conocimiento.

\begin{tabular}{lllll}
\hline \multicolumn{5}{c}{ Tiempo disponible para la gestión del conocimiento } \\
\hline & $\begin{array}{l}\text { ¿Considera } \\
\text { que no tiene } \\
\text { tiempo para } \\
\text { gestionar el } \\
\text { conocimiento? }\end{array}$ & $\begin{array}{l}\text { ¿Dispondría } \\
\text { de hasta } 5 \\
\text { horas a la } \\
\text { semana? }\end{array}$ & $\begin{array}{l}\text { ¿Dispondría } \\
\text { de hasta } 10 \\
\text { horas a la } \\
\text { semana? }\end{array}$ & $\begin{array}{l}\text { ¿Dispondría } \\
\text { de más de } \\
10 \text { horas a } \\
\text { la semana? }\end{array}$ \\
& & & & \\
& $8.8 \%$ & $\mathbf{3 4 . 8 \%}$ & $8.7 \%$ & $8.7 \%$ \\
Totalmente de acuerdo & $13.0 \%$ & $26.1 \%$ & $\mathbf{3 0 . 4 \%}$ & $21.7 \%$ \\
De acuerdo & $21.7 \%$ & $21.7 \%$ & $17.5 \%$ & $21.7 \%$ \\
Ni de acuerdo, ni en desacuerdo & $21.7 \%$ & $8.7 \%$ & $21.7 \%$ & $\mathbf{2 6 . 2 \%}$ \\
En desacuerdo & $\mathbf{3 4 . 8 \%}$ & $8.7 \%$ & $21.7 \%$ & $21.7 \%$ \\
Totalmente en desacuerdo & $100.0 \%$ & $100.0 \%$ & $100.0 \%$ & $100.0 \%$ \\
& & &
\end{tabular}

Se rescató que el $56.5 \%$ está en de acuerdo con que no tiene tiempo, el $60.9 \%$ dedicaría hasta 5 horas a la semana, el $43.4 \%$ está en desacuerdo en invertir hasta 10 horas y el $47.9 \%$ está en de acuerdo en invertir más de 10 horas en la gestión del conocimiento. 


\section{Conclusiones y trabajos futuros}

Los desarrolladores están de acuerdo en compartir el conocimiento dentro de su organización y ámbito. Prefieren compartir el conocimiento con sus compañeros y luego hacia el exterior de la organización. Consideran que el conocimiento tiene un alto valor para sí mismos, su organización la industria y el país. Consideran que el conocimiento es personal, pero que le pertenece a la organización. Desconocen y les resulta compleja la conversión del conocimiento tácito a explícito. Están convencidos de que el conocimiento se puede reutilizar y que aprender de otros permite mayor productividad a la organización. Que están dispuestos a compartir el conocimiento algunos sin pago, pero en otros esperan reconocimientos, bonos y pagos. Que les interesa y consultan el conocimiento de otros aun cuando no haya una política de la organización que así lo determine. Para la transferencia del conocimiento prefieren un SGC y en segundo lugar cursos, manuales y videos. Que cuentan con sus propios repositorios de conocimiento y que los comparten. Que están dispuestas a dedicar hasta cinco horas para la GC.

Se emite una propuesta de recomendaciones para las MiyPEDS para considerar práctica de la GC y la necesidad de un SGC.

- Incluir procesos de GC en su empresa.

- Sensibilizar a los desarrolladores para fomentar la GC.

- Capacitar a los desarrolladores para manejar el conocimiento con seguridad.

- Definir un método y técnicas para la conversión de conocimiento tácito a explícito.

- Contar con un SGC para captar, almacenar y compartir el conocimiento.

- Crear incentivos monetarios y no monetarios para fomentar la GC.

- Medir el impacto de la GC una vez implementada.

Esta investigación se desprende de otra, de la cual se ha creado un modelo que ya se ha publicado; también se está trabajando en una plataforma de un SGC para implementarse en MiyPEDS con el fin de probar el modelo, hacer los ajustes necesarios para proponer se valore su uso en las organizaciones que desarrollan software en México.

De acuerdo a los resultados los trabajadores del conocimiento de las MiyPEDS practican y muestran disposición para llevar a cabo la Gestión del Conocimiento. Por lo anterior es más importante sensibilizar de la necesidad de considerar esta actividad como importante e incorporarla en sus procesos de dichas organizaciones. Ante tal escenario existen interrogantes como: ¿Cuál es la prospectiva de los directivos de las MiyPEDS acerca de la Gestión del Conocimiento? ¿Modificar políticas fomentará la Gestión del conocimiento? ¿Incluir la gestión del conocimiento como un proceso más hará más productivas a las MiyPEDS? ¿Se logrará cambiar la cultura de la Gestión del Conocimiento en las MiyPEDS? Para encontrar estas incógnitas se continuará con esta investigación.

Finalmente se pretenden integrar técnicas de inteligencia artificial en el SGC con el fin de hacerlo más efectivo. 


\section{Referencias}

1. Capote, J., Llanten, J., Pardo, C., González, A., Collazos, C.: Gestión del conocimiento como apoyo para la mejora de procesos software en las micro, pequeñas y medianas empresas. Revista ingeniería e investigación, 28(1), 137-145 (2008)

2. Collison, C., Parcell, G.: La gestión del conocimiento, Lecciones prácticas de una empresa líder. Buenos Aires: Paidós (2003)

3. Davenport, T., Prusak, L.: Conocimiento en acción, Como las organizaciones manejan lo que saben. Buenos Aires: Prentice Hall (2001)

4. INEGI. Instituto Nacional de Geografia y Estadística. Recuperado el 10 de 06 de 2013, de http://www3.inegi.org.mx/sistemas/mapa/denue/default.aspx

5. Maldonado, G., Martínez, M. D., García, R.: La influencia de la gestión del conocimiento en el nivel de competitividad de las PyMEs manufactureras de Aguascalientes. Investigación y Ciencia (55), 24-31 (2012)

6. Martínez, Martínez, A., Corrales, M.: Administración de conocimiento y desarrollo basado en conocimiento, redes e innovación. México D. F.: CENGAGE Learning (2010)

7. Nonaka, S., Takeuchi, N.: La organización creadora del conocimiento. México: Oxford (1999)

8. Normalización y Certificación Electrónica A. C. Guía práctica de implantación de los requisitos de la NMX-I-059-NYCE-2005 (MoPRoSoft). México D. F.: Normalización y Certificación Electrónica, A. C. (2007)

9. NYCE A.C. Guía práctica de implantación de los requisitos de la NMX-I-059-NYCE-2005 (MoProSoft) (Primera ed.). México D. F.: Normalización y Certificación A. C. (2007)

10. Ruggles, R., Holtshouse, D.: La ventaja del conocimiento. México: CECSA (1999)

11. Ruiz, S., Ledeneva, Y., Morales, R.: Base de conocimiento de los procesos de desarrollo de software a través de un modelo de un sistema de gestión del conocimiento. Research in Computing Science, 55, 113-123 (2012)

12. Santiago, K., Laureano, A. L., Sánchez, J., Sarmiento, E., Domínguez, O.: Sistema basado en conocimiento para la industria del vestido en México. Research in computing science, 55, 151-162 (2012)

13. Valhondo, D.: Gestión del conocimiento: del mito a la realidad. Madrid: Ediciones Díaz de Santos (2003)

14. Velasco, A.: ¿Cómo pueden las ciencias, las técnicas, las artes y las humanidades contribuir a la democracia, la libertad y la equidad? Ciencia, Revista de la Academia Mexicana de Ciencias, 64(1), 10-17 (2013) 